\title{
PUBLICACIONES ESPAÑOLAS DE HISTORIA DE LA CIENCIA DIFUNDIDAS A TRAVES DE INTERNET
}

A lo largo de 1995 han aparecido dos publicaciones sobre Historia de la Ciencia cuya difusión se realiza exclusivamente a través de Internet: Conecta y Antilia.

\section{CONECTA \\ Boletín de Noticias de Historia de la Ciencia, la Medicina y la Tecnología}

CONECTA es un boletín de noticias sobre Historia de la Ciencia, la Medicina y la Tecnología que comenzó a funcionar en mayo de 1995 con el objetivo de distribuir noticias e informaciones de utilidad para los profesionales e investigadores de estas áreas de conocimiento, en principio del Estado español, pero también de la comunidad latinoamericana y con atención al entorno europeo.

En los números hasta ahora distribuidos más o menos cada quince días, aunque su periodicidad es irregular, han aparecido noticias sobre libros, congresos, conferencias, y sobre recursos de Internet que puedan resultar de utilidad. Se pretende contar con reseñas de libros, informaciones sobre la labor docente de los diferentes departamentos universitarios, proyectos de investigación en marcha, tesis doctorales en curso de realización y preguntas, cuestiones e informaciones de interés en general.

Pretende por tanto ser un vehículo de comunicación rápido, sin otras pretensiones que la de actuar como nexo de unión entre la comunidad de historiadores de la ciencia, la medicina y la tecnología.

El boletín se distribuye a través del correo electrónico pero no funciona como una lista de distribución en la que cualquier mensaje enviado se redistribuye automáticamente a todos los miembros de la lista, sino que las informaciones se van acumulando y son distribuidas en un solo envío cada diez o quince días.

Para recibirlo basta enviar un mensaje expresando tal deseo a:

quique@vm.cpd.ua.es

Todas las colaboraciones han de enviarse, asimismo, a esta dirección electrónica, y animamos a todos los interesados a hacerlo.

Enrique Perdiguero

División de Historia de la Ciencia

UNIVERSIDAD DE ALICANTE (ESPAÑA)

quique@vm.cpd.ua.es 


\section{Antilia \\ Revista Española de Historia de las Ciencias de la Naturaleza y la Tecnología Spanish Journal of History of Natural Sciences and Technology}

En los últimos días de diciembre de 1995 hizo su aparición Antilia, una revista dedicada a Historia de la Ciencias de la Naturaleza y de la Tecnología en España y países del área latinoamericana. Para favorecer el conocimiento en el mundo anglosajón de la producción histórico científica española y latinoamericana, la revista consta de dos versiones, una en español y otra en inglés.

Antilia pretende ser una publicación periódica equiparable a cualquier revista académica de formato tradicional, y consta de las secciones habituales de trabajos originales, reseñas de libros y una sección de notas y noticias.

Antilia no precisa de subscripción o pago de inscripción alguna: el lector interesado no debe contar más que con un ordenador personal, acceso a Internet y algún programa para visualizar WWW. Otra posibilidad para acceder, sacrificando no obstante calidad de presentación, es mediante el programa lynx, para visualización en modo texto de páginas WWW. El acceso a Antilia es, por tanto, libre y gratuito, en la dirección http://www.ucm.es/OTROS/antilia.

El primer volumen contó con la colaboración de Silvia Figueiroa con un artículo sobre las Escuelas de Ingeniería como «loci» institucionales de las Ciencias Geológicas en Brasil durante el siglo XIX, otro de Antonio González Bueno y Raúl Rodríguez Nozal, sobre Conocimiento científico y poder en la España Ilustrada: hacia la supremacía comercial a través de la Botánica medicinal, un tercero de Juan Francisco López Sánchez, Manuel Valera Candel y Carlos López Fernández sobre La Academia de Guardias Marinas de Cartagena (1776-1824); y finalmente un trabajo de Francisco Pelayo López sobre La conexión terrestre entre Cuba y el continente americano: una alternativa paleontológica a la deriva continental. Se incluían además dos breves reseñas bibliográficas sobre una reciente edición de la Historia Naturalde Cayo Plinio y la autobiografía de Irene Claremont de Castillejo.

Información adicional sobre Antilia puede solicitarse a:

Joaquin Fernández - Alfredo Baratas

Historia de la Biología

Facultad de Biología

Universidad Complutense de Madrid

28940 Madrid

Tel. (91) 39449 82. Fax: (91) 3944981

e-mail: antilia@eucmax.sim.ucm.es 